\title{
Avaliação biofarmacotécnica in vitro de formas farmacêuticas sólidas contendo doxiciclina
}

\author{
Geysa Aguiar', Luciane Gomes Faria², Humberto Gomes Ferraz², Cristina Helena do Reis Serra², \\ Valentina Porta ${ }^{2^{*}}$ \\ 1 Departamento de Farmácia, Centro de Ciências da Saúde, Universidade de Fortaleza (UNIFOR), \\ ${ }^{2}$ Laboratório de Biofarmacotécnica (BIOFAR), Departamento de Farmácia, Faculdade de Ciências Farmacêuticas, \\ Universidade de São Paulo
}

* Correspondência:

V. Porta

Depto. de Farmácia

FCF/USP

Av. Prof. Lineu Prestes, 580 - Bloco 13 05389-970 - Cidade Universitária

São Paulo - SP - Brasil

E-mail:vporta@usp.br
A absorção de fármacos a partir de formas farmacêuticas sólidas, administradas por via oral, depende de sua liberação, da dissolução ou solubilização dos mesmos em condições fisiológicas e da permeabilidade das membranas do trato gastrintestinal. Portanto, a dissolução in vitro é fundamental para se prever o desempenho in vivo do fármaco. Pretendeu-se neste trabalho, realizar avaliação biofarmacotécnica in vitro através de testes fisico-químicos e avaliação da cinética e eficiência de dissolução de quatro lotes de duas formulações do mercado nacional contendo $100 \mathrm{mg}$ de doxiciclina. Utilizou-se o método descrito pela Farmacopéia Americana para realização do ensaio de dissolução. A análise da cinética de dissolução foi avaliada por meio dos parâmetros $k_{s}$ (constante de velocidade de dissolução) e $t_{50 \%}$ (tempo necessário para dissolução de $50 \%$ do fármaco presente na forma farmacêutica). Calculou-se, também, a eficiência de dissolução (ED\%). De acordo com os resultados obtidos, verificou-se que todas as amostras avaliadas apresentaram-se de acordo com os compêndios oficiais no que se referiu a peso médio, teor de fármaco, uniformidade de conteúdo e teste de dissolução. Em relação à cinética de dissolução observou-se que todos os produtos apresentaram cinética de primeira ordem. Para a eficiência de dissolução encontraram-se valores entre 58,48\% e 78,39\%.

\section{INTRODUÇÃO}

A absorção de fármacos, a partir de formas farmacêuticas sólidas administradas por via oral, depende de sua liberação e dissolução nas condições fisiológicas e permeabilidade das membranas biológicas que necessitam transpor (Brasil, 2003c; United States, 1997). Com base nestas considerações, pode-se afirmar que a dissolução in vitro é uma etapa relevante para prever o desempenho in vivo do medicamento. 
Qualquer fator que altere os processos de desagregação e dissolução poderá afetar diretamente a biodisponibilidade, expressa em termos de quantidade de fármaco absorvido e velocidade do processo de absorção (Storpirtis et al., 1999).

O perfil de dissolução relaciona a porcentagem de fármaco dissolvido em função do tempo (Abdou, 1989) e representa uma técnica relativamente rápida e barata para avaliar formas farmacêuticas sólidas antes do teste clínico. Permite, também, a obtenção de parâmetros cinéticos, que são importantes para determinar a velocidade e eficiência do processo, além do tempo necessário para que determinadas porcentagens do fármaco se dissolvam, possibilitando, desta forma, conclusões a respeito das características biofarmacotécnicas in vitro de determinada formulação (Storpirtis, 1999).

Assim, embora a comparação definitiva entre formulações requeira o teste de bioequivalência em humanos, é prudente realizar uma extensa comparação in vitro, incluindo dados de dissolução, para maximizar as chances de sucesso de bioequivalência (Moore, Flanner, 1996).

Diversos produtos à base de doxiciclina, classificados como similares, estão presentes no mercado brasileiro de medicamentos e são, muitas vezes, utilizados de forma intercambiável com o medicamento de referência ou inovador. Com base nestas considerações, torna-se interessante realizar avaliações biofarmacotécnicas in vitro de formulações do mercado nacional contendo doxiciclina uma vez que os resultados podem fornecer indicações sobre o desempenho in vivo desses medicamentos.

O presente trabalho tem como objetivo validar um método analítico para quantificação in vitro de doxiciclina e avaliar as características biofarmacotécnicas de amostras de drágeas e comprimidos revestidos do antibiótico provenientes de lotes de diferentes fabricantes, enfatizando-se a avaliação da cinética e eficiência de dissolução.

\section{MATERIAL E MÉTODOS}

Foram avaliados os lotes 00457002 F (R1) e 00457004 (R2) do produto referência (Vibramicina ${ }^{\circledR}$ - Laboratório Pfizer Ltda: drágeas contendo $100 \mathrm{mg}$ de doxiciclina) e os lotes 7500 (T1) e 994644 (T2) do produto teste (Legrand Doxiciclina ${ }^{\circledR}$ - EMS Indústria Farmacêutica Ltda: comprimidos revestidos contendo $100 \mathrm{mg}$ de doxiciclina). Todas as análises foram realizadas utilizando-se padrão secundário de doxiciclina hiclato fornecido por EMS Indústria Farmacêutica Ltda.

Para a determinação do teor de doxiciclina nas formulações foram utilizados metanol grau analítico (Merck), água desmineralizada, balança analítica Mettler Toledo (modelo AB204-5), centrífuga Heraeus, (modelo Megafure 2.0), banho ultrasônico Odontobrás (modelo Cleaner 284D) e espectrofotômetro Vankel (modelo Cary 50 UV/VIS). Para o ensaio de dissolução, foram utilizados água desgaseificada, e aparelho de dissolução Vankel (modelo VK 7010), equipado com seis cubas e coletor automático de amostras, acoplado a espectrofotômetro Vankel (modelo Cary 50 UV/VIS).

Para determinação dos teores de doxiciclina, foram preparadas soluções aquosas padrão e amostra, submetendo-as à leitura em espectrofotômetro, utilizando comprimento de onda de $276 \mathrm{~nm}$ e água desmineralizada como branco. Utilizou-se, no cálculo, o peso médio de cada formulação e o teor de princípio ativo foi expresso pela média de três determinações.

No preparo da solução padrão, pesaram-se exatamente, cerca de $50 \mathrm{mg}$ de doxiciclina (padrão de referência) e transferiram-se para balão volumétrico de $50 \mathrm{~mL}$. Adicionaram-se cerca de $25 \mathrm{~mL}$ de metanol p.a. e procedeu-se à agitação até completa dissolução, completandose, posteriormente, o volume com metanol p.a. Em seguida, retirou-se uma alíquota de $0,5 \mathrm{~mL}$ a qual foi diluida com água desmineralizada em balão volumétrico de $50 \mathrm{~mL}$.

Para o preparo da solução amostra, trituraram-se 10 comprimidos ou drágeas de doxiciclina e pesou-se exatamente uma quantidade de pó equivalente a cerca do peso médio do comprimido, transferindo-se para balão volumétrico de $100 \mathrm{~mL}$. Acrescentaram-se cerca de $50 \mathrm{~mL}$ de água desmineralizada e procedeu-se à solubilização durante 10 minutos em banho ultrassônico, completando-se, a seguir, o volume com água. $10 \mathrm{~mL}$ desta solução foram centrifugados a 2000 rpm por 10 minutos; diluiu-se, posteriormente, $0,5 \mathrm{~mL}$ do sobrenadante com água em balão volumétrico de $50 \mathrm{~mL}$.

A validação do método baseou-se em critérios estabelecidos pela Resolução n ${ }^{\circ} .899$ e Farmacopéia Americana (Brasil, 2003b; United States, 2005) através da determinação dos parâmetros de especificidade, limite de quantificação, linearidade, exatidão e precisão.

Verificou-se a especificidade do método através da análise do espectro de absorção de amostra placebo, observando-se a existência ou não de interferência com o espectro de absorção da doxiciclina no comprimento de onda de $276 \mathrm{~nm}$. O limite de quantificação foi determinado com base no ruído do espectrofotômetro e confirmado através da análise repetitiva de amostras contendo doxiciclina na concentração correspondente ao limite de quantificação. A linearidade foi estabelecida através da construção da curva de calibração com soluções padrão de doxiciclina em concentrações que variaram de 1,0 a 90,0 Pg/mL. 
A exatidão foi determinada conforme método descrito pela AOAC International (1995), avaliando-se a concentração de doxiciclina resultante após adição de quantidades crescentes de padrão às soluções amostra, enquanto que a precisão foi determinada através da análise repetitiva (sextuplicata) de amostras de doxiciclina preparadas no mesmo dia (precisão intra-dia) e em dias diferentes (precisão inter-dias) sendo expressa pelo desvio padrão relativo dos resultados destas análises.

Os ensaios de peso médio e uniformidade de conteúdo foram realizados como descrito pela Farmacopéia Brasileira (1996).

O ensaio de dissolução foi realizado utilizando-se água degaseificada como meio de dissolução, volume de $900 \mathrm{~mL}$, aparato 2 (pá) com altura de $4,5 \mathrm{~cm}$ do fundo da cuba, rotação de $75 \mathrm{rpm}$ e temperatura de $37^{\circ} \mathrm{C} \pm 0,5^{\circ} \mathrm{C}$. Realizaram-se coletas automáticas nos tempos de: $3,6,9$, $12,15,30,45$ e 90 minutos, para obter o perfil de dissolução. A concentração do fármaco nas amostras foi determinada por espectrofotometria em comprimento de onda de 276 nm (United States, 2005).

Para o estudo da cinética de dissolução, foram aplicados os modelos de zero ordem, traçando-se gráficos de quantidade de fármaco não dissolvido versus tempo, e de primeira ordem, através de gráficos do logaritmo neperiano $(\ln )$ da quantidade de fármaco não dissolvido versus tempo (El Yazigi, 1981). Outros parâmetros relativos à cinética de dissolução como constante de velocidade de dissolução $\left(\mathrm{k}_{\mathrm{s}}\right)$ e meia-vida de dissolução $\left(\mathrm{t}_{50 \%}\right)$ também foram determinados.

A eficiência de dissolução (ED) foi calculada a partir das curvas de porcentagem de fármaco dissolvido versus tempo como descrito por Khan (1975). A comparação entre os valores de ED dos quatro produtos testados foi realizada por meio de análise de variância (ANOVA).

\section{RESULTADOS}

A validação do método espectrofotométrico para quantificação de doxiciclina nas análises do teor de quimioterápico, uniformidade de conteúdo e nos ensaio de dissolução apresentou resultados dentro das especificações exigidas pela Resolução n. 899 (Brasil, 2003b). O espectro de absorção obtido para doxiciclina, em solução aquosa, revelou pico no comprimento de onda de $274,9 \mathrm{~nm}$ bastante próximo ao descrito pela Farmacopéia Americana (United States, 2005), que é $276 \mathrm{~nm}$. Optou-se pela utilização deste último no desenvolvimento e validação do método analítico para quantificação de doxiciclina em comprimidos e em meio de dissolução. A Tabela I apresenta os resultados da validação do método espectrofotométrico para quantificação de doxiciclina e a Figura 1 os espectros de absorção de doxiciclina em solução aquosa na concentração de $20 \mu \mathrm{g} / \mathrm{mL}$ e do placebo, respectivamente. O coeficiente de determinação $\left(\mathrm{R}^{2}\right)$ da reta de calibração foi de 0,9998 .

TABELA I - Resultados da validação do método espectrofotométrico para quantificação de doxiciclina

\begin{tabular}{lc}
\hline $\begin{array}{l}\text { Parâmetro } \\
\text { de validação }\end{array}$ & $\begin{array}{c}\text { Método } \\
\text { espectrofotométrico }\end{array}$ \\
\hline Especificidade & $\begin{array}{c}\text { Ausência de interferentes } \\
\text { Limite de quantificação }\end{array}$ \\
Linearidade & $1,0 \mu \mathrm{g} / \mathrm{mL}$ \\
Exatidão & $1,0 \mu \mathrm{g} / \mathrm{mL}$ a $90,0 \mu \mathrm{g} / \mathrm{mL}$ \\
Precisão intra-dia & $100,3 \%$ a $102,4 \%$ \\
Precisão inter-dias & $0,57 \%$ \\
\hline
\end{tabular}

A Tabela II apresenta os resultados médios dos testes de peso médio e teor do quimioterápico com seus res-

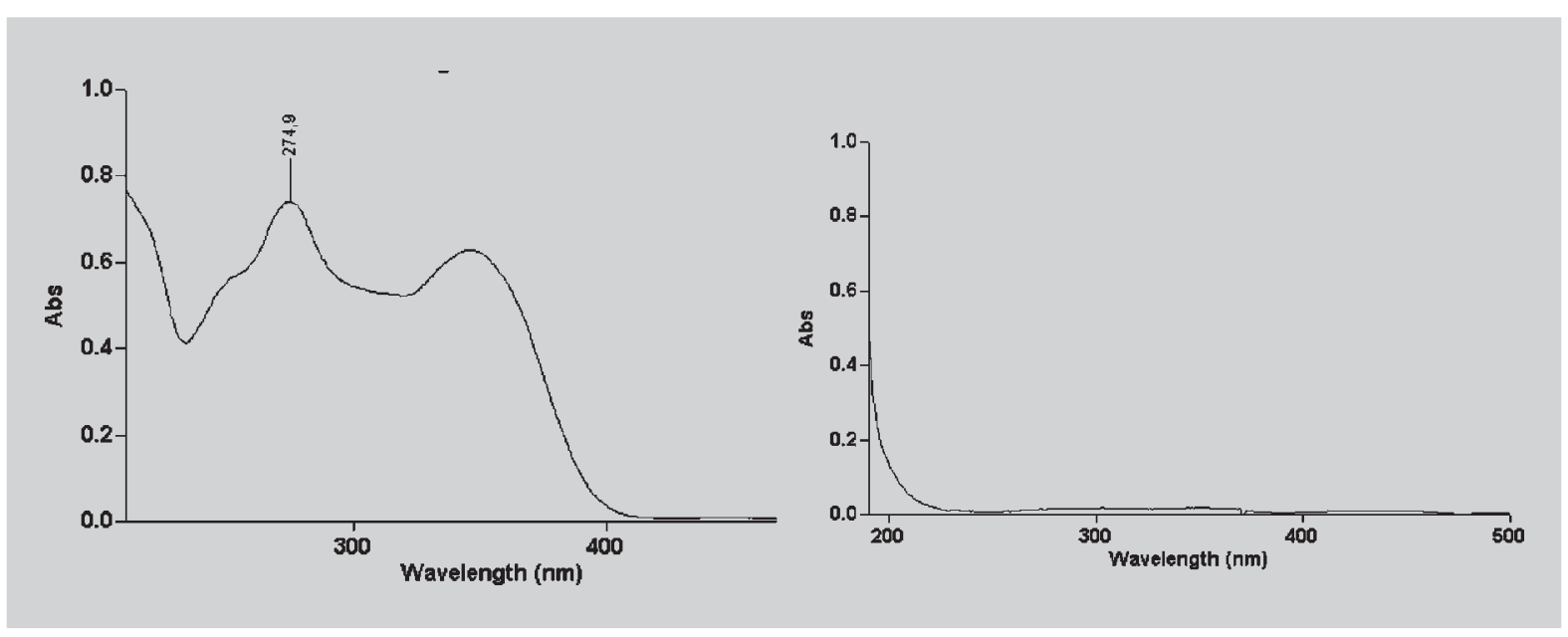

FIGURA 1 - Espectros de absorção de doxiciclina em solução aquosa $(20 \mu \mathrm{g} / \mathrm{mL})$ e do placebo (à direita). 
TABELA II - Resultados dos testes de peso médio $(n=20)$ e teor do antibiótico $(n=3)$ dos produtos referência $(R 1$ e R2) e teste (T1 e T2)

\begin{tabular}{lcccc}
\hline Ensaio & R1 & R2 & T1 & T2 \\
\hline Peso médio (mg) & $257,13(1,76)$ & $257,88(1,72)$ & $364,53(1,34)$ & $367,71(0,89)$ \\
Teor do antibiótico (\%) & $99,93(1,08)$ & $100,01(2,92)$ & $102,59(1,24)$ & $98,50(1,90)$ \\
\hline
\end{tabular}

Os valores entre parênteses indicam o desvio padrão relativo de cada ensaio.

pectivos desvios padrão relativos e a Tabela III mostra o teor do composto em cada amostra analisada no teste de uniformidade de conteúdo.

O comportamento de dissolução do composto, nas condições experimentais adotadas está ilustrado, comparativamente, na Figura 2.

As Figuras 3 e 4 apresentam as retas resultantes da aplicação dos modelos matemáticos de ordem zero e de primeira ordem aos resultados médios da dissolução dos quatro lotes testados e a Tabela IV mostra os respectivos coeficientes de determinação $\left(\mathrm{R}^{2}\right)$. Foram incluídos os pontos correspondentes aos tempos de 0 a 45 minutos uma vez que, após 45 minutos, observa-se a presença de um patamar nos perfis de dissolução, indicando o término do processo. Com base nos valores obtidos para o coeficiente de determinação $\left(\mathrm{R}^{2}\right)$, verificou-se que o modelo de cinética de primeira ordem aplica-se melhor aos resultados obtidos que o modelo de ordem zero.

Com base nos parâmetros obtidos pela cinética de primeira ordem, calculou-se a constante de velocidade de
TABELA III - Resultados do teste de uniformidade de conteúdo de princípio ativo em amostras dos produtos referência (R1 e R2) e teste (T1 e T2). DP = desvio padrão; DPR $=$ desvio padrão relativo

\begin{tabular}{ccccc}
\hline Amostra & $\begin{array}{c}\text { R1 } \\
\mathbf{( \% )}\end{array}$ & $\begin{array}{c}\mathbf{R 2} \\
\mathbf{( \% )}\end{array}$ & $\begin{array}{c}\text { T1 } \\
\mathbf{( \% )}\end{array}$ & $\begin{array}{c}\text { T2 } \\
\mathbf{( \% )}\end{array}$ \\
\hline 1 & 94,25 & 94,42 & 104,40 & 109,76 \\
2 & 98,98 & 101,38 & 100,54 & 103,50 \\
3 & 101,12 & 105,01 & 107,21 & 108,34 \\
4 & 104,66 & 98,08 & 107,70 & 107,76 \\
5 & 103,93 & 101,38 & 108,17 & 109,65 \\
6 & 103,70 & 98,60 & 114,78 & 97,90 \\
7 & 107,24 & 98,98 & 102,31 & 108,89 \\
8 & 94,08 & 94,86 & 101,27 & 105,50 \\
9 & 100,60 & 103,88 & 99,85 & 105,50 \\
10 & 100,43 & 114,89 & 109,73 & 105,44 \\
\hline Média & 100,90 & 101,15 & 105,59 & 106,22 \\
DP & 4,30 & 5,93 & 4,77 & 3,60 \\
DPR (\%) & 4,26 & 5,87 & 4,52 & 3,39 \\
\hline
\end{tabular}

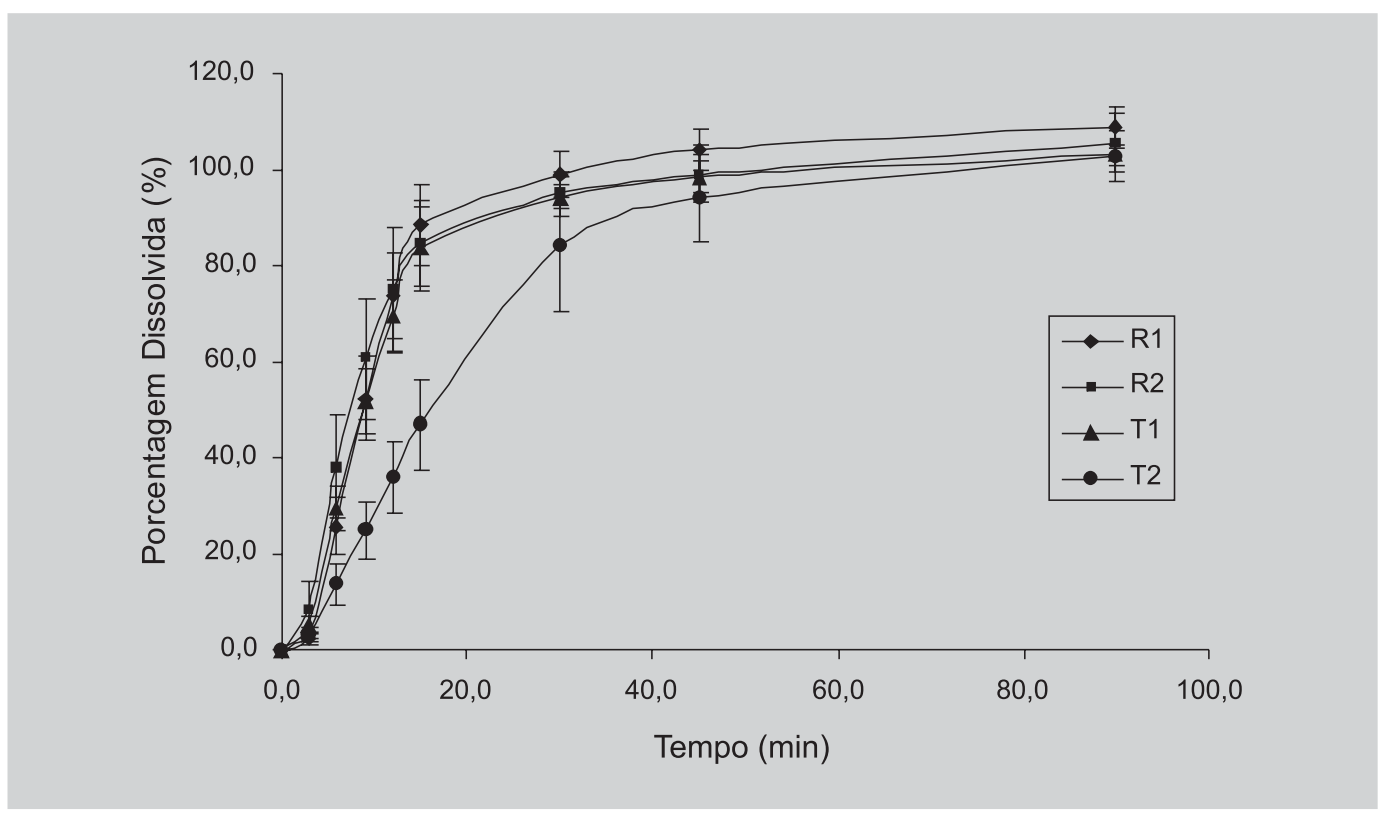

FIGURA 2 - Porcentagem média de doxiciclina dissolvida (\%) em função do tempo, ao se submeter os produtos referência (R1 e R2) e teste (T1 e T2) à dissolução em água degaseificada (75 rpm; aparato $\left.2 ; 37^{\circ} \mathrm{C}\right)$. As barras verticais correspondem ao desvio padrão. 


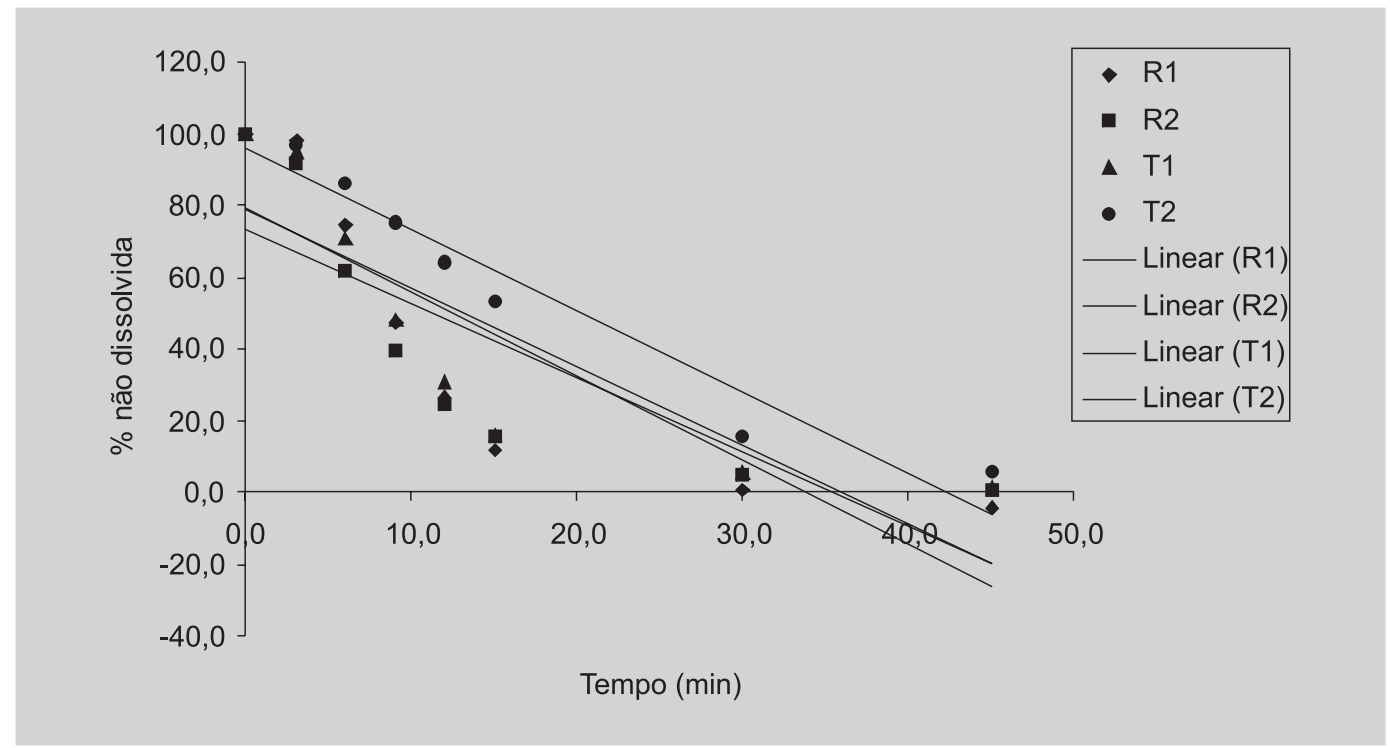

FIGURA 3 - Porcentagem média de doxiciclina não dissolvida (\%) em função do tempo, ao se submeter os produtos referência (R1 e R2) e teste (T1 e T2) à dissolução em água degaseificada (75 rpm, aparato 2; $\left.37^{\circ} \mathrm{C}\right)$. Aplicação do modelo de ordem zero.

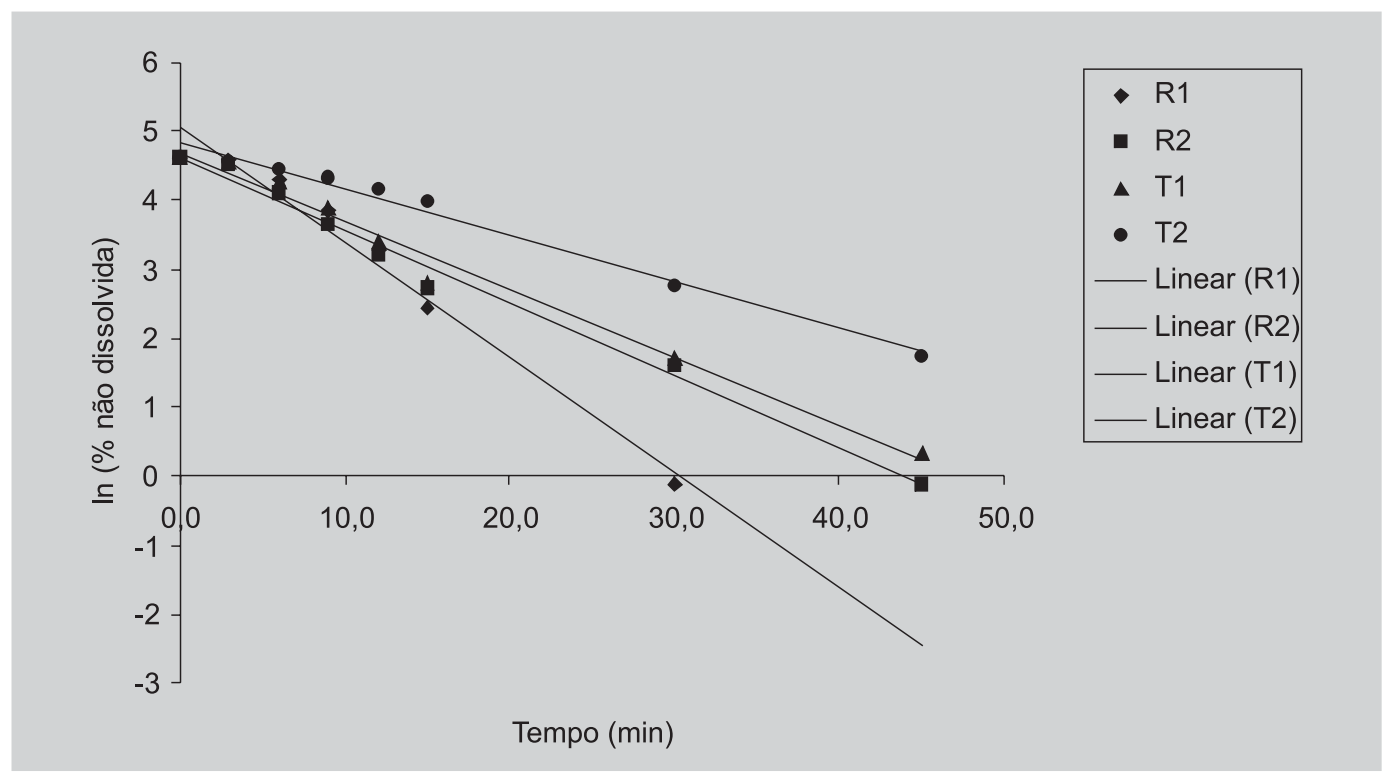

FIGURA 4 - Transformação logarítmica da porcentagem média de doxiciclina não dissolvida (\%) em função do tempo, ao se submeter os produtos referência (R1 e R2) e teste (T1 e T2) à dissolução em água degaseificada (75 rpm; aparato $2 ; 37^{\circ} \mathrm{C}$ ). Aplicação do modelo de primeira ordem.

dissolução $\left(\mathrm{k}_{\mathrm{s}}\right)$ e o tempo médio necessário para dissolução de $50 \%\left(\mathrm{t}_{50 \%}\right)$ da doxiciclina presente nos comprimidos revestidos e drágeas dos lotes avaliados (Tabela V).

As Tabelas VI e VII apresentam, respectivamente, os valores médios de eficiência de dissolução dos quatro produtos testados e o resultado da análise de variância (ANOVA). Verifica-se que existe diferença significativa entre $\mathrm{T} 2$ e todos os outros produtos testados e entre $\mathrm{T} 1 \mathrm{e}$
TABELA IV - Coeficientes de determinação $\left(\mathrm{R}^{2}\right)$ relativos às retas resultantes da aplicação dos modelos matemáticos de ordem zero e de primeira ordem aos resultados médios da dissolução dos produtos referência e teste

\begin{tabular}{lcccc}
\hline Modelo & R1 & R2 & T1 & T2 \\
\hline Ordem zero & 0,7203 & 0,6778 & 0,7215 & 0,9453 \\
Primeira ordem & 0,9742 & 0,9887 & 0,9847 & 0,9851 \\
\hline
\end{tabular}


R1, mas não existe diferença significativa entre T1 e R2 e entre R1 e R2.

TABELA V - Valores de constante de velocidade de dissolução $\left(\mathrm{k}_{\mathrm{s}}\right)$ e tempo médio necessário para dissolução de $50 \%$ de doxiciclina $\left(\mathrm{t}_{50 \%}\right)$ para os produtos referência (R1 e R2) e teste (T1 e T2) após submetê-los à dissolução em água desgaseificada ( $75 \mathrm{rpm}$; aparato $2 ; 37^{\circ} \mathrm{C}$ )

\begin{tabular}{lcccc}
\hline Parâmetro & R1 & R2 & T1 & T2 \\
\hline $\mathrm{k}\left(\mathrm{min}^{-1}\right)$ & 0,1675 & 0,1058 & 0,0990 & 0,0673 \\
$\mathrm{t}_{50 \%}(\mathrm{~min})$ & 4,14 & 6,55 & 7,00 & 10,30 \\
\hline
\end{tabular}

TABELA VI - Eficiência de dissolução de doxiciclina presente nos produtos referência (R1 e R2) e teste (T1 e T2) ao submetê-los à dissolução em água desgaseificada (75 rpm; aparato $2 ; 37^{\circ} \mathrm{C}$ )

\begin{tabular}{lcccc}
\hline Parâmetro & R1 & R2 & T1 & T2 \\
\hline ED (\%) & 78,39 & 77,33 & 75,07 & 58,48 \\
\hline Os var
\end{tabular}

Os valores representam a média de doze determinações

TABELA VII - Resultados da análise de variância da eficiência de dissolução de doxiciclina presente nos produtos referência (R1 e R2) e teste (T1 e T2) ao submetê-los à dissolução em água desgaseificada (75 rpm; aparato $\left.2 ; 37^{\circ} \mathrm{C}\right) \cdot \mathrm{p}=$ nível de significância

\begin{tabular}{lccc}
\hline & \multicolumn{3}{c}{ nível de significância (p) } \\
& $\mathbf{R} 1$ & $\mathbf{R 2}$ & T1 \\
\hline $\mathrm{R} 2$ & 0,07276 & & \\
$\mathrm{~T} 1$ & 0,003354 & 0,132642 & \\
$\mathrm{~T} 2$ & $1,25 \times 10^{-5}$ & 0,000109 & 0,000718 \\
\hline
\end{tabular}

$\mathrm{p}<0,05$ indica diferença significativa.

\section{DISCUSSÃO}

O método desenvolvido mostrou-se apropriado para quantificação de doxiciclina em comprimidos revestidos e drágeas. Obtiveram-se resultados adequados em relação aos parâmetros de validação: especificidade, linearidade, limite de quantificação, precisão e exatidão conforme a Resolução n. 899 (Brasil, 2003b) e Farmacopéia Americana (United States, 2005).

O pico de absorção obtido para solução aquosa de doxiciclina foi em $274,9 \mathrm{~nm}$, não sendo observada qualquer influência dos excipientes empregados nos picos de absorção deste fármaco. Neste estudo, utilizou-se comprimen- to de onda de $276 \mathrm{~nm}$ que é o preconizado pela Farmacopéia Americana (United States, 2005).

A reta de calibração demonstra linearidade em concentrações de $1,0 \mathrm{mg} / \mathrm{mL}$ a $90,0 \mathrm{mg} / \mathrm{mL}$, podendo-se, portanto, trabalhar nestes limites de concentração. O limite de quantificação foi de $1,0 \mathrm{mg} / \mathrm{mL}$ com precisão de $7,55 \%$ e exatidão de 106,00\%, valores aceitáveis de acordo com os limites estabelecidos pela ANVISA (Brasil, 2003b).

Verificou-se, através da análise do espectro de absorção do placebo, que não há interferência com a doxiciclina no comprimento de onda utilizado $(276 \mathrm{~nm})$, comprovando-se a especificidade do método.

Analisando-se os resultados de variação de peso dos comprimidos revestidos e das drágeas (Tabela II) observou-se que os valores dos desvios padrão relativos foram baixos, variando de $0,89 \%$ a $1,76 \%$. Tomando como base os critérios recomendados pelas Farmacopéias Brasileira e Americana (Farmacopéia, 1996; United States, 2005), os quatro lotes estudados encontram-se dentro dos limites especificados.

O teor de fármaco dos lotes analisados variou entre $98,50 \%$ e $102,59 \%$. Os coeficientes de variação apresentaram valores entre $1,08 \%$ e $2,92 \%$. Os resultados referentes ao teor de doxiciclina dos produtos analisados, obtidos a partir da análise espectrofotométrica, encontram-se em conformidade com o preconizado em compêndios oficiais, cuja especificação é de 90 a $110 \%$ do valor rotulado (Farmacopéia, 1996; United States, 2005).

Segundo critérios recomendados pela Farmacopéia Americana (United States, 2005), o teor do fármaco em cada uma das 10 unidades analisadas deve estar entre $85 \%$ e $115 \%$ do valor estipulado na embalagem e o desvio padrão relativo deve ser menor ou igual a $6 \%$. Os resultados dos testes de uniformidade de conteúdo dos lotes analisados estão dentro dos limites aceitáveis com valores médios de $100,90 \%, 101,15 \%, 105,59 \%$ e $106,22 \%$ e valores de desvio padrão relativo de 4,26\%, 5,87\%, 4,52\% e 3,39\% para R1, R2, T1 e T2, respectivamente.

A avaliação dos perfis de dissolução (Figura 2) obtidos a partir dos produtos em estudo indica que os produtos referência R1 e R2 são semelhantes, mostrando que a formulação, independentemente do lote, apresenta-se bastante homogênea. Por outro lado, a visualização dos perfis de dissolução dos lotes do produto teste sugere que, dependendo do lote, os perfis apresentaram-se de forma diferenciada, indicando possíveis problemas de produção e/ou qualidade das matérias-primas empregadas para fabricação de cada um deles. Apesar disto, todos os lotes estudados foram aprovados em relação ao ensaio de dissolução, em que não menos de $85 \%$ do valor rotulado de doxiciclina deve dissolver em 90 minutos (Brasil, 2003c; 
United States, 2005). Em relação ao perfil de dissolução observou-se que os produtos que mais se assemelharam foram referência $\mathrm{R} 2$ e teste $\mathrm{T} 1$.

O estudo da cinética do processo de dissolução, empregando-se os modelos de zero e primeira ordem, foi efetuado para os produtos analisados. Através da linearização das curvas de dissolução, foram obtidos os respectivos coeficientes de determinação $\left(\mathrm{R}^{2}\right)$. Observouse que o modelo mais adequado, isto é, aquele que forneceu maiores valores de $\mathrm{R}^{2}$, foi o de primeira ordem para todos os produtos, o que é esperado para formas farmacêuticas sólidas de liberação convencional (Ishi et al., 1996).

Embora os valores de ks (constante de velocidade de dissolução) não possam ser utilizados para comparação direta, uma vez que os mesmos variam consideravelmente entre os modelos cinéticos, sua importância está no fato de que são empregados nos cálculos de $\mathrm{t}_{50 \%}$ (meia vida de dissolução). Este último é de extrema importância, na medida em que se estabelece o tempo necessário para que $50 \%$ do fármaco esteja dissolvido. Assim, para produtos nos quais a liberação do fármaco é mais rápida, os valores de $\mathrm{t}_{50 \%}$ serão mais baixos. Verifica-se que, para este parâmetro, todos os lotes analisados apresentaram valores baixos, de até, aproximadamente, 10 minutos. Esta observação sugere que em um curto espaço de tempo, a forma farmacêutica libera metade da quantidade do fármaco contido, fato este, realmente adequado para uma forma farmacêutica de liberação imediata. Os produtos R2 e T1 apresentaram valores de $\mathrm{t}_{50 \%}$ mais próximos, de 6,56 e 7,01 minutos, respectivamente.

A eficiência de dissolução (ED\%) também permite comparar adequadamente os perfis de dissolução de fármacos (Khan, 1975). Esta variável está relacionada com a quantidade real de fármaco que se encontra dissolvida no meio e, desta forma, pode-se ter um melhor prognóstico dos resultados in vivo.

Em relação à ED\%, o medicamento de referência R2 não apresentou diferença significativa com R1 e T1, o que não pôde ser observado entre R1 e T1 e com o produto teste $\mathrm{T} 2$, que foi considerado diferente estatisticamente dos demais. Os lotes que mostraram um melhor desempenho na eficiência de dissolução foram R1 e R2.

Os produtos avaliados no presente estudo são classificados, segundo a ANVISA (Brasil, 2003a) e Food and Drug Administration (United States, 1997) como equivalentes farmacêuticos sendo, portanto, comercializados no Brasil como similares intercambiáveis. Neste caso, espera-se que sejam equivalentes em termos de eficácia clínica e segurança (Storpirtis, 1999).

A classificação do produto referência como drágea e do produto teste como comprimido revestido pode gerar dúvidas a respeito da equivalência farmacêutica entre ambos. Na verdade, podemos considerar a drágea como um tipo particular de comprimido revestido em que este revestimento é composto de açúcar e não por polímero. Atualmente, os revestimentos açucarados vêm sendo cada vez mais substituídos por polímeros, que cumprem com a mesma função de proteção, mascaramento de sabor e odor desagradáveis e/ou melhoria no aspecto do comprimido, e apresentam menos dificuldades de produção (Ansel et al., 1995).

Apesar dos quatro lotes dos dois produtos analisados cumprirem com as especificações de qualidade, caracterizando, efetivamente, a equivalência farmacêutica entre eles, observou-se que o perfil e a eficiência de dissolução do produto T2 apresentou comportamento diferenciado dos demais. Portanto, a realização de estudo in vivo mostra-se necessária para conclusões definitivas sobre biodisponibilidade e bioequivalência dos produtos em estudo.

\section{CONCLUSÃO}

O método analítico desenvolvido mostrou-se apropriado para quantificação in vitro de doxiciclina em comprimidos revestidos e drágeas, obtendo-se resultados adequados para os parâmetros de validação. Os lotes testados apresentaram peso médio, teor de fármaco e uniformidade de conteúdo de acordo com os critérios farmacopéicos, sendo também aprovados no ensaio de dissolução, tendo $85 \%$ do fármaco dissolvido em menos de 90 minutos.

O estudo da cinética de dissolução das amostras testadas indicou que o modelo mais adequado foi o de primeira ordem para produtos analisados e, baseando-se nos parâmetros da cinética de dissolução $\left(\mathrm{k}_{\mathrm{s}}\right.$ e $\left.\mathrm{t}_{50 \%}\right)$ e na eficiência de dissolução (ED), observou-se que os produtos R1, R2 e T1 apresentaram perfis semelhantes em relação às porcentagens de liberação do fármaco. Apesar disto, a realização de estudo in vivo mostra-se necessária para conclusões definitivas sobre biodisponibilidade e bioequivalência dos produtos em estudo.

\section{ABSTRACT}

\section{In vitro biopharmaceutical evaluation of solid dosage forms containing doxycycline}

The process of drug absorption from solid dosage forms following oral administration depends on drug delivery from dosage form, its dissolution in gastrointestinal conditions and permeation through the intestinal membrane. Therefore, in vitro dissolution is very important to predict the in vivo performance of a drug 
contained in a solid dosage form. The purpose of this study was to perform an in vitro biopharmaceutical evaluation, through physicochemical, dissolution kinetics and dissolution efficacy tests of four batches of two commercial products available in the Brazilian market, containing $100 \mathrm{mg}$ of doxycycline. The dissolution method described in the United States Pharmacopeia (USP) was used for the dissolution kinetics analysis. It was evaluated by the parameters $k_{s}$ (dissolution rate constant), $t_{50 \%}$ (time to promote the dissolution of $50 \%$ of the drug in the pharmaceutical dosage form) and dissolution efficacy. According to the results it was verified that all the batches were in accordance with official specifications for average weight, doxyxycline content, uniformity content, and dissolution tests. All produtcts showed first order dissolution kinetics. Dissolution efficacy values ranged from $58,48 \%$ to $78,39 \%$.

UNITERMS: Doxycycline. Biopharmaceutics. Dissolution kinetics. Bioequivalence. Generic drug products.

\section{REFERÊNCIAS BIBLIOGRAFICAS}

ABDOU, H.M. Dissolution, bioavailability and bioequivalence. Easton: Mack Printing, 1989. 554p.

ANSEL, H.C., POPOVICH, N.G., ALLEN, L.V. Pharmaceutical dosage forms and drug delivery systems. 6.ed. Baltimore: Williams \& Wilkins, 1995. p.6198.

ASSOCIATION OF OFFICIAL ANALYTICAL CHEMISTS. Officcial methods of analysis of AOAC international. 16.ed. Arlington: AOAC, 1995. v.1, pt.1, p.xviii.

BRASIL. Resolução RDC n.135, de 29 de maio de 2003. A Agência Nacional de Vigilância Sanitária aprova o regulamento técnico para medicamentos genéricos. Diário Oficial da União, Brasília, 2003a. Disponível em: $<$ http://www.anvisa.gov.br>. Acesso em: 15 abr. 2004.

BRASIL. Resolução RE n.899, de 29 de maio de 2003. A Agência Nacional de Vigilância Sanitária aprova guia para validação de métodos analíticos. Diário Oficial da União, Brasília, 2003b. Disponível em: <http:// www.anvisa.gov.br>.. Acesso em: 15 abr. 2004.
BRASIL. Resolução RE n.901, de 29 de maio de 2003. A Agência Nacional de Vigilância Sanitária aprova guia para ensaios de dissolução para formas farmacêuticas sólidas de liberação imediata (FFSLI). Diário Oficial da União, Brasília, 2003c. Disponível em: <http:// www.anvisa.gov.br>. Acesso em: 15 abr. 2004.

EL-YAZIGI, A. Disintegration: dissolution analysis of percent dissolved time data. J. Pharm. Sci., New York, v.70, n.5, p.535-537, 1981

FARMACOPÉIABrasileira. 4.ed. São Paulo: Atheneu, 1996. pt.II. fasc. 1. cap. V.1.6.

ISHI, K., SAITOU, R., YAMADA, R., ITAI, S., NEMOTO, $\mathrm{M}$. Novel approach determination of correlation between in vivo and in vitro dissolution using the optimization technique. Chem. Pharm. Bull., Tokyo, v.44, n.8, p.15501555, 1996.

KHAN, K.A. The concept of dissolution efficiency. $J$. Pharm. Pharmacol., Wallingford, v.27, p.48-49, 1975.

MOORE, J.W., FLANNER, H.H. Mathematical comparison of dissolution profiles. Pharm. Techn., Duluth, v.20, n.6, p.64-74, 1996.

STORPIRTIS, S. Biofarmacotécnica: fundamentos de biodisponibilidade, bioequivalência, dissolução e intercambialidade de medicamentos genéricos. São Paulo: s.n, 1999. 78p.

STORPIRTIS, S., OLIVEIRA, P.G., RODRÍGUEZ, D. MARANHO, D. Considerações biofarmacotécnicas relevantes na fabricação de medicamentos genéricos: fatores que afetam a dissolução e a absorção de fármacos. Rev. Bras. Cienc. Farm., São Paulo, v.35, n.1, p.1-16, 1999.

UNITED States Pharmacopeia: 28 ed. Rockville: United States Pharmacopeial Convention, 2005.

UNITED STATES. Departament of Healthy and Human Services. Food and Drug Administration. Center for Drug Evaluation and Research. Guidance for industry: dissolution testing of immediate release solid oral dosage forms. Rockville: CMC, 1997. 17p. Disponível em: $<$ http://www.fda.gov/cder/guidance/1713bp1.pdf $>$. Acesso em: 4 out. 2004.

Recebido para publicação em 27 de setembro de 2004. Aceito para publicação em 24 de fevereiro de 2005. 